\title{
Der Übergang als Reibung und Unbestimmtheit
}

\author{
,Kulturinteraktion als produktives Modell \\ für die interkulturelle Literaturwissenschaft
}

Miriam Llamas Ubieto

\begin{abstract}
Based on an interactionalist and praxeological cultural model, interaction is inherent in cultural phenomena and cultural practice. For this reason, this article will show what factors must occur so that interaction can be described as intercultural. This article will also show how interaction works internally. From the perspective of this theoretical proposal, such interaction or friction (Tsing) of schemas is considered to be a transition, both temporally and materially. There are two contradictory and productive aspects inherent in this transition. The two aspects are: 1. the creation of regulatory boundaries and 2. the vagueness, questioning or even the transformation that can occur in relation to said boundaries. Through literary examples, it will be explained how the aforementioned interactions, which are specific to transition, are structured literarily through concrete textual strategies. Focusing on the interaction and the interdisciplinary combination of cultural theories (Kogge, Reckwitz, Waldenfels) and methods based on discourse and literature (Foucault, Bakhtin) this article will clarify the relation between intra- and extra-textual, collective and individual texts and discourses so that an approach for a new reading of literary texts can be presented.
\end{abstract}

Title: Transition as Friction and Indeterminacy: >Cultural Interaction< as a Productive Model for Intercultural Literary Studies

Keywords: transition; interculturality and literature; friction of schemes; interaction; Haitti Chérie

\section{EINFÜHRUNG}

Seit Jahrzehnten gibt es in der Komparatistik und in der Literaturwissenschaft die Tendenz, die Frage der kulturellen Begegnung und der Übergangsprozesse aus zwei unterschiedlichen Perspektiven zu betrachten: aus der der Alterität auf der einen Seite und aus der des Aufeinanderprallens und der Überschneidung statischer, homogener und schon zuvor bestehender Entitäten und Grenzen andererseits. Demzufolge zeigen diese Annäherungen an die Texte zwei Schwierigkeiten: Erstens, sie kategorisieren die Texte anhand von Kriterien der kulturellen Differenzierung, die man auf die Texte projiziert, bevor man die textuelle Konfiguration an sich in Betracht zieht; und zweitens, sie gehen nicht der Interaktion an sich auf den Grund. Im Gegensatz dazu ist die Interaktion dem kulturellen Phänomen und der kulturellen Praxis inhärent, wenn man ein interaktionistisches und praxeologisches Kulturmodell als Ansatzpunkt annimmt. 


\section{Die Kulturinteraktion}

Der hier vorgeschlagene Ansatz stützt sich auf ein Kulturkonzept, das eine Lösung zwischen der holistischen Ebene der kollektiven Diskurse und der partikulär-individuellen Ebene annimmt. Das heißt, es stellt die Verbindung zwischen der Ebene der Diskurse oder Ordnungen und der Ebene der konkreten individuellen Interaktionsbeziehung her, die wiederkehrende Schemata untereinander ins Verhältnis setzt. Den Zusammenfluss beider Ebenen (objektiv und subjektiv) stellen die kulturellen Praktiken dar, den Voraussetzungen der Theorie der »Sozialen Praktiken« von Andreas Reckwitz (vgl. 2000: 542-588) folgend. Es ist zu beachten, dass diese geteilten Schemata außerhalb der Praktiken des Subjekts nicht selbstständig existieren, sondern sich ihre Existenz und Bildung in Diskursen und Ordnungen aus ihrer Wiederkehr in besagten individuellen und konkreten Praktiken des Handelns und Interpretierens ableitet (vgl. ebd.: 559).

In dem Moment, in dem >etwas < das Subjekt anspricht und mit den Schemata in seinem Inneren in Friktion gerät oder bewirkt, dass diese sich aneinander reiben, entsteht, nach der Hermeneutik des Nichtverstehens von Werner Kogge (vgl. 2002: 262-265), ein Moment des Zweifels. Dieser Raum der Unbestimmtheit dauert an, bis das Subjekt die richtige Art, sich zu verhalten, gefunden hat oder dieser Situation Sinn zuschreiben kann, also eine passende Antwort findet. Erst dann tritt die Differenz zwischen Schemata und deren Zuschreibung zu kollektiven Ordnungen in Erscheinung, aber paradoxerweise ist dies auch der Moment, in dem besagte Differenz überschritten werden kann. In diesen internen Prozessen der symbolischen Entstehung drängt der Aspekt der Friktion (vgl. Tsing 2005), relational und kreativ, in den Vordergrund, der in dem auftritt, was ich als >Intervall< bezeichne.

Damit dieser Prozess als interkulturell verstanden wird, fehlt nur noch, dass die Schemata bei ihrer Zuschreibung als Teil kollektiver Ordnungen als untereinander kulturell inkompatibel gekennzeichnet werden. Zu diesem Zweck treten zwei Figuren auf den Plan, die zwischen diesem ansprechenden >etwas < und den zu benutzenden Schemata vermitteln: die Figur des Dritten und die Dimension der Fremderfahrung. Wie der Phänomenologe Bernhard Waldenfels (vgl. 2000) aufzeigt, nimmt das Subjekt die Rolle eines Dritten an, eine Instanz, die dann entsteht, wenn wir miteinander interagieren, und die die Regelungen in Gang setzt, um >etwas Sinn zuzuschreiben, um es zu erkennen und es gegenüber einem anderen als >etwas $<$ zu kategorisieren. Ihre Funktion ist deswegen in diesem bestimmten Moment auch die des Begründers von Ordnungen. Aber über diese kategoriale Dimension hinaus tritt der fremde Anspruch auch als Fremderfahrung in Erscheinung, als Unvereinbarkeit mit den vertrauten verinnerlichten Schemata: Es handelt sich demnach also um eine Dimension, die sich auf die praktische Identität des Subjekts bezieht. ${ }^{1}$

1 | Ich folge in diesem Sinne der Unterscheidung von Patrick C. Hogan (vgl. 2004: 8) zwischen kategorialer und praktischer Identität. 
Dieser Prozess, an dessen Ende eine Antwort gegeben wird, kann als Übergang verstanden werden. Im materiell-symbolischen Sinne impliziert der Anspruch von >etwas $<$ an das Subjekt bereits eine Interaktion und das Auftreten einer Grenze, die paradoxerweise gleichzeitig überschritten wird, da der Anspruch in das Subjekt eindringt. Des Weiteren ereignet sich zwischen den sich reibenden Schemata im Inneren des Subjekts, die es verwendet, um eine Antwort zu geben, das gleiche relationale Paradoxon. Aber dieser Übergang besitzt auch eine zeitliche Dimension, die für die Entstehung jeder neuen Antwort notwendig ist. Das bedeutet, diese Bewegung der Schemata ereignet sich in einem konkreten Moment der Unbestimmtheit und führt zu einem neuen zeitlichen Raum der partikulären und einzigartigen Antwort, obwohl sie sich wiederkehrender Schemata bedient.

Sogar die einfachste und automatischste Reaktualisierung der Schemata, die andere aufhebt, die mit ihr in Konflikt treten würden, z.B. in Form einer Projizierung des Eigenen auf das Fremde, beinhaltet die paradoxe Erfahrung des Entstehens und gleichzeitigen Überschreitens von Grenzen. Da das Ziehen einer Grenze bekanntermaßen einen Moment außerhalb der Ordnung mit sich bringt, folglich auch einen Moment des Überschreitens (vgl. Foucault 1994: 237) oder des Übergangs in das Unerreichbare, ist demnach dieses andere, an das man grenzt, nicht a priori festgelegt, sondern es ist relational, temporär und situationsbedingt.

\section{Die Dialogizität als Grundlage der Interaktionen UND DES TEXTES}

Die Fiktion wird zur privilegierten Urheberin, welche die Praktiken der kulturellen Interaktion sichtbar macht und diese Übergänge, die normalerweise während der Praktiken im Inneren des Subjekts verborgen bleiben, transkodifiziert. Letzten Endes gehört der Text weder exklusiv dem Autoren-Ich noch einem anderen (einer anderen Welt), sondern dem Interaktionsintervall zwischen den beiden. Und die Interaktion wird sichtbar, da besagter Text schließlich aus der Externalisierung oder Erweiterung, nicht des Ichs oder des Objekts, sondern eben des Interaktionsintervalls besteht, in dem sich die Rekombinationen der Schemata ereignen, was üblicherweise verborgen bleibt.

Auf diese Weise benutzt der künstlerische Text als fiktives Objekt des Übergangs zwischen dem Inneren des Subjekts und dem Äußeren seine simulierenden und transformierenden Eigenschaften. So erlangt alles den gleichen fiktiven Status und die Unterschiede zwischen den Ordnungen liegen nun nicht mehr im realen, kategorisierenden Selbst eines Subjekts (eines Autors) gegenüber dem, was ihm fremd ist, sondern in den internen fiktiven Abgrenzungen und Strategien des einzelnen Textes selbst, welche die Brücke zum diskursiven Supratextuellen schlagen.

Der Dialogizitätsbegriff von Bachtin ermöglicht es, die Charakteristiken des Interaktionsintervalls zwischen den Schemata der Ordnungen in den Texten zu 
erkunden. Seine Auffassung von Sprache, Aussage und Wort als sozioideologische Ereignisse, die am dialogischen Leben teilhaben (vgl. Bachtin 1984: 293), ist vergleichbar mit der von den Praktiken, da sich bei ihnen wie in den Aussagen die kollektive Ebene mit der partikulär-individuellen anhand der Interaktionen (erste dialogische Dimension) verbindet, und die einen (Aussagen) und die anderen (Praktiken) schöpfen ihren Sinn oder ihr Handeln aus den Zwischenbeziehungen der Schemata der Diskurse und Sprachen, die Teil dieser Interaktion sind (zweite dialogische Dimension oder Interdiskursivität).

Bekanntermaßen (vgl. Bachtin 1971: 266) ist dank dieser dialogischen Beziehung der Aussage mit Elementen, die außerhalb von ihr stehen (und die sie gleichzeitig bilden), die dialogische Theorie eine der ersten, die die kollektivdiskursive Ebene mit der linguistisch-literarischen im engeren Sinne verbindet. Aus diesem Grund wird sie zur notwendigen Brücke für die Verbindung von Diskurs und Text, diesen als partikulär-individuelle Äußerung der kollektiven Diskurse verstehend, die sie eingliedert und gleichzeitig hilft zu konstruieren, wie Schlieben-Lange (vgl. 1995: 6) anmerkt.

Die sozialen Sprachen oder kollektiven Diskurse verhalten sich in der Aussage relational (genau wie in der kulturellen Praktik), aber allzu oft vergessen die systemischen Interpretationen der Dialogizität etwas, worauf Bachtin seinerzeit bereits hinwies: und zwar, dass diese Beziehungen sich in einer partikulär-individuellen Aussage verkörpern, die von einem Sender ausgeht, dessen Standpunkt sie in der symbolischen Materie ausdrückt (vgl. Bachtin 1971: 269). Daraus folgt erstens: Ohne die Verankerung der subjektiven Ebene des Textes, das heißt ohne die partikuläre dialogische Interaktion, die der Text verflicht, treten die sozialen Sprachen oder Ordnungen weder hervor noch sind sie auszumachen. Und zweitens: Diese Verbindungen zwischen verkörperten Standpunkten, die in den Aussagen zweier Subjekte vorkommen oder im Inneren des bivokalen Wortes, gehören zu einer bestimmten Art: der dialogischen. Ich zitiere:

"Das Leben ist schön". "Das Leben ist schön". Hier haben wir zwei völlig identische Urteile, eigentlich also ein einziges Urteil, das wir zweimal geschrieben (oder ausgesprochen) haben, aber dieses "zweimal " bezieht sich nur auf die sprachliche Realisierung, nicht auf das Urteil selbst. Zwar können wir auch hier von dem logischen Verhältnis der Identität zwischen zwei Urteilen sprechen. Aber erst wenn dieses Urteil in zwei verschiedenen Äußerungen zwei [sic] verschiedener Subjekte ausgesprochen wird, entstehen zwischen diesen Äußerungen dialogische Beziehungen (der Zustimmung, der Bestätigung). (Bachtin 1971: 204f.; Hervorh. i.0.)

Bachtin interessiert sich für das Geschehen der Interaktion und der Aussage, die als Teil dieses Geschehens die Spannung, den Kampf und die Gegenüberstellung erhält. Die dialogische Interaktion ist eine relationale Form der »wechselseitigen Erhellung« (Bachtin 1979: 293) zwischen zwei Entgegnungen, »sie führen gleichsam ein Gespräch miteinander« (ebd.: 213).

Obwohl also die Äußerung ein neuer Akt oder ein neues Produkt ist, das im Sinndialog erschaffen wird, hat dieser Dialog nicht den harmonischen Konsens 
durch eine Synthese zum Ziel. Sogar derjenige, der in Form einfacher Interaktion $^{2}$ den Gegensatz oder das problematische Element beseitigt und dazu tendiert, eine Stimme oder Ideologie, Sprache oder einen Diskurs als einzig authentischen und gültigen zu verherrlichen, ist im Grunde auch dialogisch und hybrid.

In dieser Art Dialogizität ist es möglich zu erahnen, dass die Interaktion in Form von Frage-Antwort oder Anspruch-Antwort erzeugt wird. Von Anfang an entwickelt Bachtin in seinen Schriften einen ethischen Ansatz für die Untersuchung des künstlerisch-literarischen Objekts, das sich auf das für die Antwort dem anderen gegenüber verantwortliche Handeln, das heißt: auf die Intersubjektivität und Responsivität, stützt. So ist jede Aussage eine Antwort, die an anderer Stelle beginnt, wie später Waldenfels (vgl. 2000: 261) sagte; jede Aussage (da sie als Antwort fungiert) beginnt also in einer anderen unterschiedlichen Aussage. Anders ausgedrückt, verlangt der Standpunkt, der in der Aussage angenommen wird, um zu antworten, eine externe Position (Extraposition) zu der, an die er sich richtet.

Auf diese Art und Weise kann sich der im Wort konzentrierte Dialog aus Stimmen und Weltanschauungen mit gestalterischen Formen entwickeln oder verdeckt bleiben. Aber wenn die Formen der »hybriden Konstruktion « (Bachtin 1979: 195) beziehungsweise die dialogischen Reibungen der Schemata in der Aussage erkennbar sind, dann ist es möglich, das Merkmal der Fremdheit ${ }^{3}$ und die Grenze aufzudecken.

\section{Die dialogische Kulturinteraktion in den Texten}

Die Intertextualität (in eingeschränktem Sinne) beschreibt das Verhältnis zwischen einem dem Sprecher eigenen Text (Rahmen) und einem fremden Text oder nichteigenen Segment, das in diesem Rahmentext absorbiert oder von ihm angeeignet wurde, sodass eine fast statische Beziehung zwischen zwei Einheiten aufgebaut wird. Aber durch die Umwandlung dieser Beziehungen in die Bestätigung des Anderen im Einen werden sie banalisiert und die Bewegung wird außen vorgelassen (vgl. Kristeva 1974: 60). Das Problem ist, einmal mehr, die Präexistenz geschlossener Systeme als Ausgangspunkt nehmen zu wollen. Nach diesen Theorien ist es so, als würde der neue Text als geschlossen und zuvor erschaffen angesehen werden und man würde an ihm einen künstlichen, exoge-

2 | Gemeint ist eine Interaktion, in der schnell bestimmte Schemata aktualisiert werden, anstatt anderer Schemata (mit denen sie in Friktion treten), ohne dass Schwierigkeiten oder Rekombinationen von Schemata verschiedener Ordnungen auftauchen würden.

3 | Bachtin selbst bietet Klassifizierungen dieser künstlerischen Formen der Kombination aus Stimmen und Diskursen an. Ihre Typologie hängt vor allem davon ab, wie die hybride Antwort ausfällt: ob sie eine andere Stimme oder einen anderen semantischen Standpunkt stilisiert, ob sie sie berücksichtigt, ihr antwortet, sie vorwegnimmt, sie parodiert etc. (Bachtin 1971: 270-291; 1979: 192-219). 
nen Zusatz entdecken, der umgeformt und integriert worden ist. Aber die kulturell unterschiedlichen Textelemente fügen sich nicht in eine vorherige Ordnung ein, die der resultierende Text wäre, da der Text an sich vorher nicht existiert. Die Markierung oder der Kontrast eines Elements als kulturell unterschiedlich und mit vorherigen Bedeutungen beladen, erfolgt in der Erstellung jedes neuen textuellen Gebildes durch die Interaktion mit anderen Komponenten des Textes (intratextuell), die wiederum auch einen Nachklang haben. In diesem dialogischen Moment werden die >refraktierten < Diskurse und Ordnungen artikuliert. Nun wird das symbolische Material den Ordnungen zugeschrieben und die Markierung findet statt.

Die textuelle Rekombination der Schemata kann, außer dass sie Ordnungen und deren Grenzen partikulär-individuell je nach der Interaktion unter ihnen entstehen lässt, diese in Frage stellen und gleichzeitig überwinden. Auf diese Weise erhält der resultierende Text gelegentlich einen transformistischen Charakter, da die vorherige Form der Materialien, die ihn bilden, während des Prozesses der textuellen Co-Kreation übertreten, verändert und umgewandelt wird. Das heißt, das, was die Andersartigkeit eines symbolischen Materials im Text charakterisiert, ist der interaktionistische Aspekt.

Im Text ereignet sich die dialogische Interaktion der Schemata intratextuell auf ästhetisch-linguistischer und semantischer Ebene und gleichzeitig im Dialog mit der diskursiven Ebene. Diese textuellen dialogischen Praktiken werden mithilfe diverser literarischer Kompositionsmittel im Text formuliert: Stilisierung, Parodie, Dialogizität narrativer Instanzen, Dialogizität semantisch-strukturell angeordneter semantischer Standpunkte, durch semantisch hybride symbolische Formen etc.

Und es handelt sich nicht um Projektionen eines reellen Subjekts oder nur um Darstellungen mit Figuren und dem Erzähler. Die entsprechenden Vorgänge im Subjekt, die wir für die kulturellen Praktiken gesehen haben, äußern sich durch aussagende und narrative Positionen oder durch semantische Positionen von Textelementen sowie durch die Handlung der Figur des Dritten (die der Leser sein kann). Das bedeutet, die Beziehung von >etwas<, das anspricht, wird durch Strategien narratologischer, semantischer, rhetorischer Art etc. ausgedrückt. Diese verbinden und kombinieren die Schemata der Diskurse und Ordnungen und stellen so textuelle Interdiskursivität her.

Im Folgenden stelle ich ein Anwendungsmodell anhand des Romans Haït Chérie (1990) von Hans Christoph Buch vor, der beispielhaft eine Vielzahl dieser Dialogismen aufweist, die den Text auf grundlegende Weise durchdringen. Der Roman ist ein postmodernes >Potpourri< über die Geschichte der kulturellen Zwischenbeziehungen der >Alten $<$ und >Neuen $<$ Welt der letzten fünf Jahrhunderte, charakterisiert durch einen postkolonialen Blick (vgl. Lützeler 1997 und 2005). Die Schelmin, Hure und Liebesgöttlichkeit des Voodoo, Erzulie, ist die Hauptfigur, die im ersten Buch ihre Geschichte erzählt. Ihre Reisen über den Ozean und ihre Veränderungen durch den Kontakt zu den großen Männern jeder Zeit übermitteln eine Erzählung, die sie von Kolumbus zu Hitler und durch die Haitianische Revolution führt. 
Unter den diversen ästhetischen Strategien, die die interkulturellen Bewegungen formen, treten das In-Szene-Setzen und die narratologische Dialogizität sehr häufig im Roman auf, wie es die Szene des ersten Kontaktes mit den Kolonisten zeigt:

Der gekrümmte Wasserhorizont wurde von einer Mastspitze durchbrochen, dann erschien ein weißes Segel, auf das mit roter Farbe ein Andreaskreuz gemalt war, sogar der Matrose im Ausguck war deutlich zu erkennen, und ein Schiffskonvoi von drei Karavellen hielt, vom Passatwind getrieben, auf unsere Küste zu und lief in rascher Fahrt in die Lagune ein - aber damals wußte ich weder, was ein Andreaskreuz, noch was ein Schiffskonvoi oder eine Karavelle ist. Ich stand starr vor Schrecken, aber noch ehe ich Zeit gefunden hatte, meine Eltern und Geschwister zu alarmieren, legten die schwimmenden Häuser am Ufer an, Ankerketten gingen rasselnd nieder, und weiße Männer, die wie Gürteltiere ringförmige Brustpanzer trugen, wateten unter einem Wald von Lanzen, Schwertern und Fahnen an Land, wo sie vor mir auf die Knie fielen, nicht etwa, um meine nackten Füße zu küssen, sondern um ihre von der Salzluft aufgesprungenen Lippen in den feuchten Sand zu drücken. Dann pflanzten sie ein gekreuztes Holz in den Boden, während ein Priester in einem sackartigen Rock unverständliche Worte murmelte und mit den Händen seltsame Zeichen in die Luft malte. (Buch 1990: 11f.)

Hier wird symbolisch die Gründung einer Ordnung dargestellt: Oder, wie Waldenfels anmerkt: »Die Stiftung einer Ordnung ist ein Ereignis, das nicht Teil der Ordnung ist, die sie ermöglicht « (Waldenfels 2006: 65). So werden am Ende die Suche des anderen und sein Auftauchen trotz alledem sowohl für die einen als auch für die anderen zu einem Pathos und nicht etwas Geplantes; aber der Moment der Anagnorisis, genauso wie er dargestellt wird, bedeutet paradoxerweise gleichzeitig die Entdeckung des Gartens Eden und die Vertreibung aus dem Paradies. Zudem wird durch die Umkehrung des bekannten Blickwinkels das im kulturellen Gedächtnis verankerte Bild der Eroberung umgewandelt, und die Konzeption selbst der >Entdeckung < als einseitig erbaute Monovision wird angezweifelt. Die Dialogizität der Blicke stellt den beidseitigen Charakter der Begegnung wieder her.

Erzulies Blick ist der, der sich bei diesem Treffen mit den fremden Wesen reibt. Diese narratologische Dialogizität der Stimme und Perspektive von Erzulie mit dem Diskurs der Eroberung, metaphorisch dargestellt durch das Eindringen der anderen, erzeugt die kritische Neubedeutung vom aufgezwungenen und gewaltsamen interkulturellen Kontakt, der die Gefundenen in eine eigene >Unterordnung > oder eine Unterordnung innerhalb einer Ordnung umformt. Von einer Mischung aus Verwicklung (oder Wissen über das Geschehene) und Distanz ausgehend, wird ein Gewaltakt beschrieben, der Erzulie und die Ihren aus den Schemata einer fremden Ordnung charakterisiert, der im Text dialogisch die interpretierende Zuweisung gegenübergestellt wird, die zum einen die unwissende Erzulie (bärtige Männer, fremd und bedrohlich) und zum anderen die >weise und alte< Erzulie (mithilfe der Animalisierung) anwendet. 
Allerdings zeigt sich die Gründung zweier Ordnungen und einer Grenze mit hierarchischen Bedeutungen anhand der Strategie des In-Szene-Setzens. Die Bewegung der Schiffe, als Objekte des Übergangs, dringt wie ein Pathos in Erzulies Wahrnehmung ein, und in diesem Moment erscheint die Grenze, die zu einer eigenen und einer fremden Ordnung gehört. Also erst wenn die vertikale Stellung des Mastes in ihrem Blickfeld die Linie des Horizonts durchbricht, taucht diese Linie als Symbol des Endes des Vertrauten auf. Die wechselseitige Bewegung der Schiffe und des antwortenden Blicks wird durch eine Überschreitung dargestellt, die das für beide Erkennbare übersteigt und die Begrenzung jedes Einzelnen in Bezug auf den anderen sichtbar macht. Die Bewegung der Friktion zeichnet die Distanzen und baut den Raum des Übergangs auf, der kein anderer ist als das Bild des Kreuzes, gebildet durch den Mast und den Horizont. Aber ein Kreuz in Bewegung, das sich verändert, je näher die Eroberer kommen, nicht statisch ist und das, trotz der Gegenseitigkeit des Kontaktes, ihn mit der Ungleichheit des kolonialen Diskurses durchtränkt. Der doppelte Diskurs des Christentums und des dem Kreuz zugehörigen Imperiums nimmt einen Charakter von Schemata dominanter Ordnungen im Verhältnis zu jenen anderen an, (in dem Moment) als die Eroberer gewaltsam mit dem vertikalen patriarchischen Symbol in die Horizontalität der zu erobernden Welt eindringen, die typischerweise im Diskurs des Kolonisten als passiv und feminin charakterisiert wird. Dieser Akt wird symbolisch vollzogen, als die Eindringlinge nach dem Überqueren der letzten Grenze ihrer Schiffe und des Ufers mit einer Geste der performativischen Aneignung das Kreuz in den Boden rammen.

Diese künstliche Konstruktion stellt Elemente in einen Dialog, die im Text selbst verschiedenen Ordnungen zugeschrieben werden, und bezieht sich auf einen historischen Hybridismus. Die in Szene gesetzte Überschreitung führt zur Umwandlung und der >Vertreibung aus dem Paradies<, wie die bereits hybride Stimme der Protagonistin belegt.

\section{SCHLUSSFOLGERUNGEN}

Das Ergebnis ist ein hybrider oder durchkreuzter Text, ${ }^{4}$ also die räumliche Konkretisierung der besagten dialogisch kulturellen Bewegung. Aber es ist gegensätzlich zum binarischen Modell der traditionellen Intertextualität. Diese Untersuchung der Dialogizität ermöglicht das Verständnis der kulturellen Beziehungen vom Text ausgehend, von der Spezifität des Ästhetisch-Literarischen und von den Kategorisierungen der kulturellen Ordnungen oder Differenzierungen aus, die er selbst aufstellt, und nicht a priori von kultureller Zugehörigkeit und Identifikation des Textes, die nicht berücksichtigen, dass er in diesem Zwischenraum des Dialogs zwischen Kulturen entsteht.

4 | Der durchkreuzte Text wird hier als ein Ergebnis der Umwandlung aufgrund der Verflechtung von Praktiken und Schemata verstanden und nicht als das Ergebnis eines Prozesses der Überschneidung von Räumen beschrieben. 


\section{LITERATUR}

Bachtin, Michail M. (1971): Probleme der Poetik Dostoevskijs. Aus dem Russ. v. Adelheid Schramm. München.

Ders. (1979): Die Ästhetik des Wortes. Aus dem Russ. v. Rainer Grübel u. Sabine Reese. Frankfurt a.M.

Ders. (1984): Appendix II. "Toward a Reworking of the Dostoevsky Book" (1961). In: Ders.: Problems of Dostoevsky's poetics. Hg. u. übers. v. Caryl Emerson. Minneapolis/London, S. 283-302.

Buch, Hans Christoph (1990): Haïti Chérie. Roman. Frankfurt a.M.

Foucault, Michel (1994): Préface à la transgression [1963]. In: Ders.: Dits et écrits. Bd. I. Paris, S. 233-250.

Hogan, Patrick C. (2004): Empire and Poetic Voice: Cognitive and Cultural Studies of Literary Tradition and Colonialism. Albany.

Kogge, Werner (2002): Die Grenzen des Verstehens. Kultur - Differenz - Diskretion. WeiIerswist.

Kristeva, Julia (1974): La Révolution dans le langage. Paris.

Lützeler, Paul Michael (1997): Der postkoloniale Blick. Deutsche Schriftsteller berichten aus der Dritten Welt. Frankfurt a.M.

Ders. (2005): Postmoderne und postkoloniale deutschsprachige Literatur. Diskurs Analyse - Kritik. Bielefeld.

Reckwitz, Andreas (2000): Die Transformation der Kulturtheorien: Zur Entwicklung eines Theorieprogramms. Weilerswist.

Schlieben-Lange, Brigitte (1995): Kulturkonflikte in Texten. In: LiLi 25, H. 97 (= Kulturkonflikte in Texten), S. 1-21.

Tsing, Anna L. (2005): Friction: An Ethnography of Global Connection. Princeton/ Woodstock.

Waldenfels, Bernhard (2000): Zwischen den Kulturen. In: Jahrbuch Deutsch als Fremdsprache 26, S. 245-261.

Ders. (2006): Antwort auf das Fremde. In: Ders.: Grundmotive einer Phänomenologie des Fremden. Frankfurt a.M., S. 56-67. 
